\title{
D-chiro-inositol, Vitamin D, and Epigallocatechin Gallate Avoid Surgery in Females with Uterine Fibroids: Two Case Reports
}

\author{
Authors: \\ *Mario Montanino Oliva ${ }^{1,2}$ \\ 1. In vitro Fertilisation (IVF) Unit, Altamedica, Rome, Italy \\ 2. UniCamillus - Saint Camillus International University of Health Sciences, Rome, Italy \\ *Correspondence to mario.montanino@artemisia.it
}

Disclosure: $\quad$ The author has declared no conflicts of interest.

Received: $\quad 03.11 .20$

Accepted: $\quad 15.02 .21$

Keywords: $\quad$ Assisted reproductive technology (ART), D-chiro-inositol (DCI), epigallocatechin gallate (EGCG), leiomyoma, myoma, uterine fibroids, vitamin D.

Citation:

EMJ Repro Health. 2021;9[1]:95-101.

\section{Abstract}

Uterine fibroids (UF) represent the most common benign tumours in females of reproductive age, and can negatively affect fertility. Patients with UFs need to reduce the tumour size with pharmacological treatments or surgically remove the fibroid before using assisted reproductive technology (ART). On the other hand, surgery implies long waiting times before ART to avoid the risk of rupture of the uterus. Long waiting periods are often unacceptable for older individuals who want to undergo ART procedures. Unfortunately, no specific and safe treatment for UFs is currently available. Here the author reports two cases of patients with UFs and associated heavy menstrual bleeding who seek pregnancy through ART. Both underwent a daily treatment with epigallocatechin gallate, vitamin D, vitamin B6, and D-chiro-inositol for 3 months. The patients showed a volume reduction of $73.8 \%$ and $68.4 \%$, respectively. This was associated with decreased blood loss ( $42.1 \%$ and $48.7 \%$, respectively). After 3 months from the end of the treatment, both patients underwent ART procedure without the need for surgical intervention.

\section{INTRODUCTION}

Uterine fibroids (UFs), also known as leiomyomas or myomas, are gynaecological tumours typical of reproductive age. ${ }^{1}$ They rarely appear before menarche and usually regress after menopause. ${ }^{2}$ Symptoms are present in approximately $50 \%$ of patients and mainly include heavy menstrual bleeding, which could lead to anaemia in some cases. Other symptoms depend on the volume and position of the tumour and comprise dyspareunia, pelvic pain associated with a feeling of pressure in the abdominal cavity,

constipation, and urinary incontinence. The International Federation of Gynecology and Obstetrics (FIGO) classified UFs based on their location in the uterus: submucosal, pedunculate submucosal, intramural, subserosal, and five other intermediate categories. Submucosal UFs negatively affect fertility as well as intramural UFs when above $4 \mathrm{~cm}$, even without cavity distortion. On the other hand, subserosal UFs have little or no effect on fertility. ${ }^{3}$

Today, the management of fibroids in those wishing to conceive is primarily surgical. However, surgery may cause pelvic and 
intrauterine adhesions, damaging uterine integrity. For this reason, waiting 6-12 months post-surgery is generally suggested in view of using assisted reproductive technology (ART). Such delay may represent an obstacle for elderly people approaching ART, decreasing their chances to achieve and sustain pregnancy. Hence, surgical removal of fibroids for infertility must be undertaken only when there is evidence to support the improvement in pregnancy outcomes. ${ }^{4}$

Pharmacological treatments are meant to reduce the symptoms and the tumour size in preparation for surgery or, possibly, to avoid the procedure altogether. They include progestogens, androgens, aromatase inhibitors, oestrogen receptor antagonists, selective progesterone receptor modulators, and gonadotropin-releasing hormone agonists. ${ }^{5}$ While many of these are used off-label, ulipristal acetate is the only selective progesterone receptor modulator with a specific indication for the treatment of UFs. ${ }^{6}$ However, ulipristal acetate was withdrawn from the market in September 2020 because of severe side effects, including liver failure.,

Recently, vitamin D and epigallocatechin gallate (EGCG) have shown promising results against UFs both in vitro and in vivo. ${ }^{8,9}$ Vitamin $D$ deficiency has been correlated with higher risk of UF development as well as with higher UF volumes. ${ }^{10}$ Moreover, vitamin D has shown efficacy in inhibiting UF growth and in improving patients' quality of life. ${ }^{9}$ EGCG is the most abundant and biologically active catechin from green tea, accounting for at least $50 \%$ of the total catechin content in green tea leaves." Several in vitro, in vivo, and clinical studies have shown multiple EGCG anti-cancer activities, including anti-proliferative, pro-apoptotic, antiangiogenic, and anti-invasive functions. ${ }^{12}$ EGCG has shown the same activities on UFs, leading to tumour volume reduction and an improvement in the quality of life. ${ }^{8}$

UFs originate from the uterine smooth muscle tissue (myometrium), and their growth depends on oestrogen and progesterone. ${ }^{13,14}$ Worthy of note, the aromatase enzyme is over-expressed in UF tissue, allowing for autocrine production of oestradiol that facilitates tumour growth. ${ }^{13}$
D-chiro-inositol (DCl) is a cyclic polyalcohol belonging to the inositol family. Inositols are present in nine different stereoisomers, of which myo-inositol (MI) is the main abundant in nature, directly followed by $\mathrm{DCl} .^{15}$ Inositol is the precursor of inositol triphosphate, which acts as second messenger in all the pathways involving G-protein-coupled receptors such as insulin, follicular stimulating hormone, and thyroid-stimulating hormone. ${ }^{16} \mathrm{MI}$ and $\mathrm{DCl}$ result effectively in the management of hormonal and metabolic alteration of polycystic ovary syndrome thanks to their activity as insulin second messengers. ${ }^{17}$ Despite their similar molecular shape, they play different roles even when involved in the same pathways. Indeed, these two molecules act as insulin second messengers: MI driving the intracellular glucose intake while $\mathrm{DCl}$ stimulates glycogen synthesis. For this reason, their ratio is specific and regulated for each organ and tissue. ${ }^{18}$ Recently, DCl showed efficacy in downregulating, in a dose-dependent manner, the mRNA expression of the aromatase enzyme in granulosa cells. ${ }^{19}$ This effect prompted the investigation of $\mathrm{DCl}$ applications in oestrogendependent pathologies, including UFs.

Here the authours report two cases of females with UF treated with $\mathrm{DCl}$, vitamin $\mathrm{D}$, and EGCG, in order to define a novel and safe nonpharmacological treatment to avoid surgery before ART.

\section{CASE DESCRIPTION}

Two females with UF, Case 1 and Case 2, were recruited between March and September 2020 and gave their oral informed consent after the explanation of the study purpose. This study was conducted following the Ethical Principles of the Helsinki Declaration and the national laws. Both patients were seeking pregnancy through ART.

The patients underwent a treatment consisting of $150 \mathrm{mg}$ epigallocatechin gallate, 1,000 IU vitamin D, $5 \mathrm{mg}$ vitamin B6, and $50 \mathrm{mg} \mathrm{DCl}$ (Delphys ${ }^{\circledR}$ plus, Farmares Srl, Rome, Italy) once per day for 3 months. This dosage was chosen because of the previous clinical data of the study of Porcaro et al., ${ }^{25}$ which obtained significant results in reducing UF volume and controlling symptoms in the absence of side effects. Transvaginal 2D images of the uterus were obtained both in the mid-sagittal 
and transverse planes by a single observer using a Voluson ${ }^{\mathrm{TM}}$ E8 (GE Healthcare, Chicago, Illinois, USA) and a $7.5 \mathrm{MHz}$ transvaginal probe. The 3D volumetric acquisitions were obtained for each UF on the mid-sagittal plane. Measurements of the fibroid in $\mathrm{mm}$ (anterio-posterior, longitudinal, and transverse) were recorded in ViewPoint ${ }^{T M}$ (GE Healthcare). The 3D data were reviewed using SonoView Pro ${ }^{\circledR}$ (Medison Ltd., Seoul, South Korea) and UF volumes were calculated using virtual organ computed-aided analysis (VOCAL). The 3D volumetric measure was re-opened using SonoView Pro software and the outline of the UFs was traced manually using VOCAL, with six steps of rotation, $30^{\circ}$ apart. After manual tracing, the VOCAL programme automatically displayed the 3D reconstructed fibroid with its volume. The patients were evaluated at baseline, after 3 months of treatment ( $\mathrm{T} 1$ ), and at followup, performed after a further 3 months without any treatment (T2).

\section{Case 1}

A 38-year-old female seeking pregnancy was referred to the authors' fertility centre. The patient, with a BMI of $21.3 \mathrm{~kg} / \mathrm{m}^{2}$, reported an intense blood loss and transvaginal sonography showed an intramural UF with a volume of 164.3 $\mathrm{cm}^{3}$. The UF led to uterine cavity distortion and the patient was eligible for surgery in view of the ART procedure. The patient reported an increase in serum vitamin D content from baseline (16.3 ng/ $\mathrm{dL})$ to the end of the treatment $(24.1 \mathrm{ng} / \mathrm{dL})$. The vitamin $D$ serum level did not report a relevant variation at T2 $(23.8 \mathrm{ng} / \mathrm{dL})$. At baseline, the patient answered a standard semi-quantitative pictorial questionnaire for the evaluation of blood loss, ${ }^{1}$ showing an estimated blood loss of $85 \mathrm{~mL}$. Although the heavy bleeding reduced red blood cell (RBC) count, with a value of 3.8 millions/ $\mathrm{mm}^{3}$, the patient showed a normal haemoglobin ( $\mathrm{Hb}$ ) level $(12.61 \mathrm{~g} / \mathrm{dL})$. At $\mathrm{T} 1$, a $70.96 \%$ reduction of UF volume was observed $\left(47.7 \mathrm{~cm}^{3}\right)$, accompanied by a $42.1 \%$ reduction of bleeding (50 $\mathrm{mL}$ ). Despite the blood loss reduction, RBC and $\mathrm{Hb}$ did not show notable improvement, with respective values of 3.8 millions $/ \mathrm{mm}^{3}$ and $12.80 \mathrm{~g} / \mathrm{dL}$. At T2, the patient underwent further evaluation after 3 months without any treatment. The results indicated a modest volume and bleeding increase, compared to $\mathrm{T} 1$ (21.54\% and $10.00 \%$, respectively). Volume reduction and blood loss are reported in Figure 1 and Figure 2 , respectively. On the other hand, the RBC count increased to $12.81 \mathrm{~g} / \mathrm{dL}$, while the haemoglobin remained unchanged $(12.72 \mathrm{~g} / \mathrm{dL}$ ) These results are reported in Table 1. After the study period, the patient did not need surgical intervention before ART.

\section{Case Two}

A 39 year-old female seeking pregnancy was referred to the author's fertility centre. The patient, with a BMI of $23.4 \mathrm{~kg} / \mathrm{m}^{2}$, reported an intense blood loss and transvaginal sonography showed an intramural UF with a volume of 77.9 $\mathrm{cm}^{3}$. The UF led to uterine cavity distortion and the patient was eligible for surgery in view of the ART procedure. The patient reported an increase in serum vitamin $D$ content from baseline (14.2 $\mathrm{ng} / \mathrm{dL})$ to the end of treatment $(19.8 \mathrm{ng} / \mathrm{dL})$. The vitamin $D$ serum level did not report a relevant variation at T2 $(20.1 \mathrm{ng} / \mathrm{dL})$. At baseline, the patients answered a standard semi-quantitative pictorial questionnaire for the evaluation of blood loss,' showing an estimated blood loss of $92 \mathrm{~mL}$. Although heavy bleeding reduced RBC with a value of 3.25 millions $/ \mathrm{mm}^{3}$, the patient showed a normal haemoglobin level (11.1 g/dL). At $\mathrm{T} 1$, a $65.98 \%$ reduction of UF volume was observed (26.5 $\mathrm{cm}^{3}$ ), accompanied by a $47.8 \%$ reduction of bleeding $(48 \mathrm{~mL})$. Despite the blood loss reduction, $\mathrm{RBC}$ and $\mathrm{Hb}$ did not show notable improvement, with respective values of 3.75 millions $/ \mathrm{mm}^{3}$ and $11.90 \mathrm{~g} / \mathrm{dL}$. At T2, the patient underwent further evaluation after 3 months without any treatment. The results indicated a modest bleeding increase despite the absence of volume increase, compared to T1 (18\%). Volume reduction and blood loss are reported in Figure 1 and Figure 2, respectively. On the other hand, the RBC count increased to $11.93 \mathrm{~g} / \mathrm{dL}$ while the haemoglobin remained unchanged $(11.57 \mathrm{~g} / \mathrm{dL})$. The results are reported in Table 1. After the study period, the patient did not need surgical intervention before ART.

\section{DISCUSSION}

In these two cases, the combination of EGCG, vitamin $\mathrm{D}$, vitamin $\mathrm{B} 6$, and $\mathrm{DCl}$ avoided surgical intervention before undergoing ART procedure. In fact, at the end of the treatment, both patients showed an important UF volume and bleeding 
reduction, and surgery was no longer necessary. Although the period of treatment was insufficient for restoring the RBC level, a normal erythrocyte count was obtained on the second followup according to the erythropoietic capacity, which requires 12-16 weeks. In previous studies, vitamin D and EGCG, alone and in combination, showed promising results in the management of UFs. ${ }^{8}$ In the study of Ciavattini et al., ${ }^{10}$ vitamin D supplementation reduced the progression to severe symptomatology and, thus, the need for conventional surgical or medical therapy.

Table 1: Results of Case Studies 1 and 2.

\begin{tabular}{|c|c|c|c|}
\hline & Baseline & $\mathrm{T} 1$ & T2 \\
\hline \multicolumn{4}{|l|}{ Case 1} \\
\hline Volume $\left(\mathrm{cm}^{3}\right)$ & 164.3 & 47.7 & 60.8 \\
\hline Bleeding $(\mathrm{mL})$ & 85 & 50 & 55 \\
\hline Menstrual length (days) & 6.0 & 5.5 & 5.5 \\
\hline Vitamin D serum level ( $\mathrm{ng} / \mathrm{dL})$ & 16.3 & 24.1 & 23.8 \\
\hline RBC (millions/mm3) & 3.80 & 3.80 & 12.81 \\
\hline $\mathrm{Hb}(\mathrm{g} / \mathrm{dL})$ & 12.61 & 12.80 & 12.72 \\
\hline \multicolumn{4}{|l|}{ Case 2} \\
\hline Volume $\left(\mathrm{cm}^{3}\right)$ & 77.9 & 26.5 & 26.5 \\
\hline Bleeding $(\mathrm{mL})$ & 92 & 48 & 57 \\
\hline Menstrual length (days) & 7.0 & 5.0 & 5.0 \\
\hline Vitamin D serum level (ng/dL) & 14.2 & 19.8 & 20.1 \\
\hline RBC (millions $/ \mathrm{mm}^{3}$ ) & 3.25 & 3.75 & 11.90 \\
\hline $\mathrm{Hb}(\mathrm{g} / \mathrm{dL})$ & 11.10 & 11.93 & 11.57 \\
\hline
\end{tabular}

RBC: red blood cell; $\mathrm{Hb}$ : haemoglobin; T1: after 3 months of treatment; T2: follow-up performed after further 3 months without any treatment.

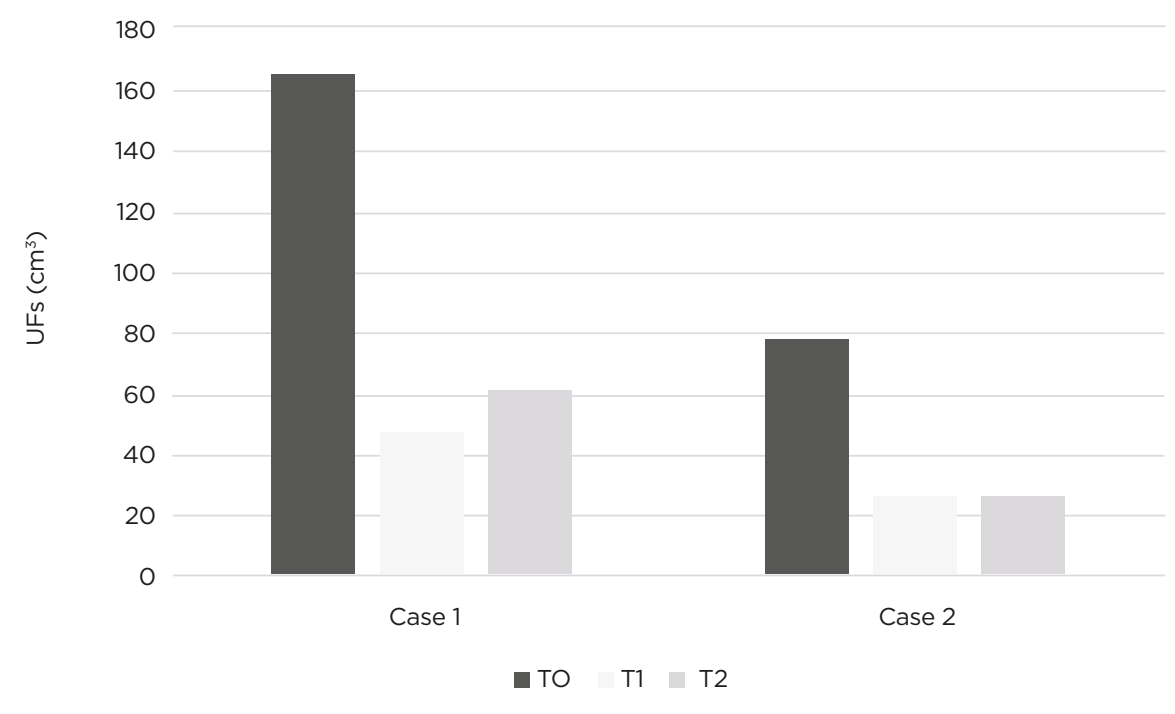

Figure 1: Uterine fibroid volume $\left(\mathrm{cm}^{3}\right)$ in Case 1 and Case 2 at baseline (TO), after the end of the treatment (T1), and after 3 months of follow-up without any treatment (T2). 


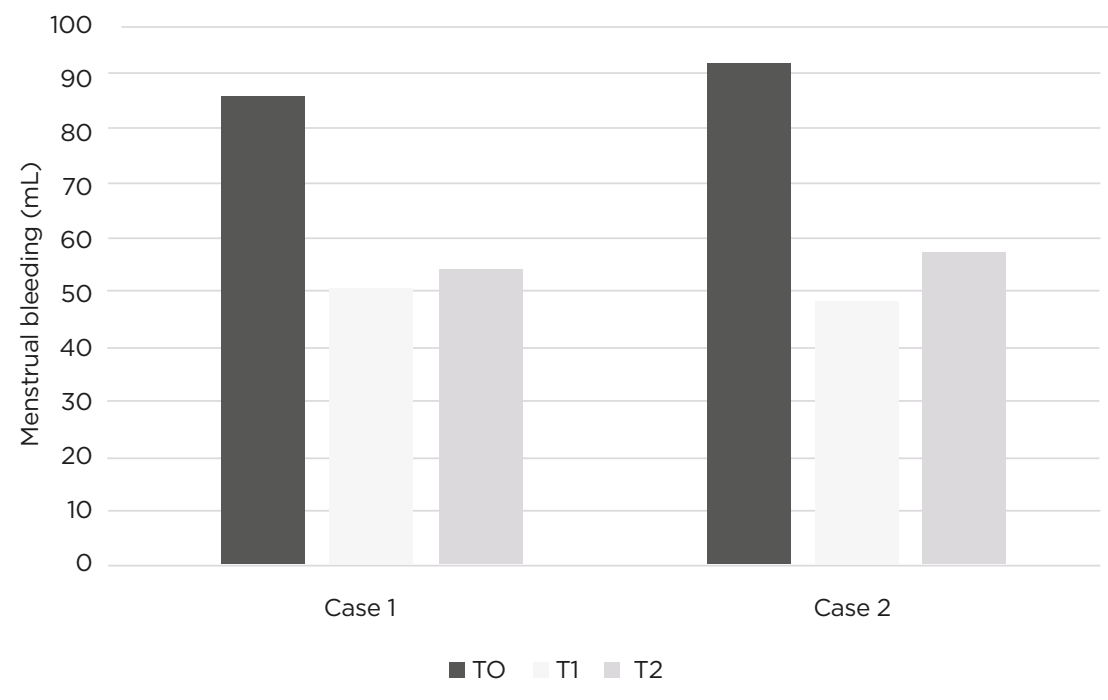

Figure 2: Menstrual bleeding ( $\mathrm{mL}$ ) in Case 1 and Case 2 at baseline (TO), after the end of the treatment (T1), and after 3 months of follow-up without any treatment (T2).

In particular, vitamin $D$ showed significant growth inhibition compared with the control, which showed an increase in UF volume as well as worsening of the symptomatology..$^{10}$ Although vitamin $D$ did not lead to UF volume reduction in the Ciavattini et al. research, recent studies report that vitamin $\mathrm{D}$ can reduce UF size both in vitro and in vivo via the suppression of cell growth and proliferation-related genes. ${ }^{20,21}$

In particular, vitamin D showed efficacy in inhibiting proliferation in UF cell line (human uterine leiomyoma cells) through the downregulation of proliferating cell nuclear antigen and cyclin-dependent kinase 1, a crucial protein involved in cell cycle regulation. In the same study, vitamin $D$ demonstrated a proapoptotic effect by reducing the expression of B-cell lymphoma $2 .{ }^{22}$

In recent years, EGCG also proved effective in the treatment of UFs., ${ }^{8,23-26}$ Its activity has been demonstrated both in vitro and in vivo, showing anti-proliferative and pro-apoptotic effects. Patients treated with oral EGCG reported UF volume reduction as well as improvement in symptomatology.

EGCG, like vitamin D, showed anti-proliferative and pro-apoptotic pathways through the downregulation of proliferating cell nuclear antigen, cyclin-dependent kinase 4, and B-cell lymphoma $2 .^{25-27}$ These effects were also demonstrated in the nude mouse model after the inoculation of the UF cell, with a significant tumour size reduction. ${ }^{26}$

In the study of Porcaro et al., ${ }^{24}$ the combination of vitamin D and EGCG has been tested for the first time in females diagnosed with UFs. The results showed UF volume reduction and improvement of the symptomatology. ${ }^{24}$

The use of aromatase inhibitors in the management of UFs resulted in a significant tumour volume and symptom reduction. ${ }^{28,29}$ However, a recent Cochrane review stated that the evidence is insufficient to support the use of aromatase inhibitor drugs in the treatment of UFs ${ }^{30}$ because of the lack of studies and the significant side effects. $\mathrm{DCl}$, in combination with $\mathrm{Ml}$ in a 40:1 ratio, enhances the signal of insulin $^{17}$ in pathologies characterised by insulin resistance, such as polycystic ovary syndrome, showing high efficacy and a good safety profile. Moreover, the effect of $\mathrm{DCl}$ in downregulating aromatase expression $^{19}$ opens to a broad spectrum of applications for different therapeutic targets, including UFs. UF cells overexpress the aromatase enzyme, producing oestradiol and sustaining their own proliferation. ${ }^{13,31}$ In this regard, this is the first report of the use of $\mathrm{DCl}$ in the management of UF before using ART. The results suggest a high efficacy in reducing UF volume and menstrual bleeding, so that surgery was no longer necessary. The activity of $\mathrm{DCl}$ 
on the aromatase might explain the important volume reduction reported in both cases. It is possible to speculate that vitamin D and EGCG exert their anti-proliferative and pro-apoptotic effects, enhanced by the activity of $\mathrm{DCl}$.

The literature reports the correlation between UFs and fertility. ${ }^{3}$ Submucosal, peduncular submucosal, and intramural UFs facing the internal uterine wall may deform the uterine cavity and alter the endometrium. As a consequence, gamete transport is impaired and the ability for embryo implantation reduced. ${ }^{3}$ Surgery should be considered in infertile patients with fibroids before using ART. $^{32}$ However, surgery is associated with a $0.47 \%$ increased risk of uterine rupture in a following pregnancy in the short-term, ${ }^{33}$ and ART procedures should be postponed. In the cases reported, treatment with $\mathrm{DCl}$, vitamin $\mathrm{D}$, and EGCG avoided surgical procedures and the need for a waiting period before ART. Randomised and controlled clinical trials are required to support these preliminary findings.

\section{CONCLUSION}

In conclusion, the combination of EGCG, vitamin $D$, and $\mathrm{DCl}$ shows high efficacy in reducing UF volume and, consequently, avoids the need for surgery. Such an approach represents an effective and safe alternative to other pharmacological treatments to avoid the delay before undergoing ART procedures but also for all those patients eligible for surgical treatment.

\section{References}

1. De La Cruz MS, Buchanan EM. Uterine fibroids: diagnosis and treatment. Am Fam Physician. 2017;95(2):100-7.

2. Englund $\mathrm{K}$ et al. Sex steroid receptors in human myometrium and fibroids: changes during the menstrual cycle and gonadotropin-releasing hormone treatment. J Clin Endocrinol Metab. 1998;83(11):4092-6.

3. Zepiridis LI et al. Infertility and uterine fibroids. Best Pract Res Clin Obstet Gynaecol. 2016;34:66-73.

4. Carranza-Mamane B et al. The management of uterine fibroids in women with otherwise unexplained infertility. J Obstet Gynaecol Can. 2015;37(3):277-85.

5. Vilos GA et al. The management of uterine leiomyomas. J Obstet Gynaecol Can. 2015;37(2):157-78.

6. Rabe T et al. Selective progesterone receptor modulators for the medical treatment of uterine fibroids with a focus on ulipristal acetate. Biomed Res Int. 2018;2018:1374821.

7. Meunier L et al. Acute liver failure requiring transplantation caused by ulipristal acetate. Clin Res Hepatol Gastroenterol. 2020;44(3):45-9.

8. Ciebiera $\mathrm{M}$ et al. The evolving role of natural compounds in the medical treatment of uterine fibroids. J Clin Med. 2020;9(5):1479.

9. Ciebiera $M$ et al. Vitamin $D$ and uterine fibroids-review of the literature and novel concepts. Int J Mol Sci. 2018;19(7):2051.

10. Ciavattini A et al. Hypovitaminosis $\mathrm{D}$ and "small burden" uterine fibroids: opportunity for a vitamin
D supplementation. Medicine (Baltimore). 2016;95(52):5698.

11. Khan $\mathrm{N}$ et al. Targeting multiple signaling pathways by green tea polyphenol (-)-epigallocatechin3-gallate. Cancer Res. 2006;66(5):2500-5.

12. Gan RY et al. Absorption, metabolism, anti-cancer effect and molecular targets of epigallocatechin gallate (EGCG): an updated review. Crit Rev Food Sci Nutr. 2018;58(6):924-41.

13. Bulun SE et al. Expression of the CYP19 gene and its product aromatase cytochrome P450 in human uterine leiomyoma tissues and cells in culture. J Clin Endocrinol Metab. 1994;78(3):736-43.

14. Ishikawa $\mathrm{H}$ et al. Progesterone is essential for maintenance and growth of uterine leiomyoma. Endocrinology. 2010;151(6):2433-42

15. Milewska EM et al. Inositol and human reproduction. From cellular metabolism to clinical use. Gynecol Endocrinol. 2016;32(9):690-5.

16. Bizzarri $M$ et al. Pharmacodynamics and pharmacokinetics of inositol(s) in health and disease. Expert Opin Drug Metab Toxicol. 2016;12(10):1181-96.

17. Genazzani AD. Inositol as putative integrative treatment for PCOS. Reprod Biomed Online. 2016;33(6):770-80.

18. Gateva A et al. The use of inositol(s) isomers in the management of polycystic ovary syndrome: a comprehensive review. Gynecol Endocrinol. 2018;34(7):545-50.

19. Sacchi $\mathrm{S}$ et al. Modulation of gonadotrophin induced steroidogenic enzymes in granulosa cells by d-chiroinositol. Reprod Biol Endocrinol. 2016;14(1):52.

20. Halder SK et al. 1,25-dihydroxyvitamin D3 treatment shrinks uterine leiomyoma tumors in the Eker rat model. Biol Reprod. 2012;86(4):116.

21. Halder SK et al. Paricalcitol, a vitamin $\mathrm{D}$ receptor activator, inhibits tumor formation in a murine model of uterine fibroids. Reprod Sci. 2014;21(9):1108-19.

22. Sharan $C$ et al. Vitamin $D$ inhibits proliferation of human uterine leiomyoma cells via catechol-Omethyltransferase. Fertil Steril. 2011:95(1):247-53.

23. Ciebiera $\mathrm{M}$ et al. Alternative oral agents in prophylaxis and therapy of uterine fibroids-an up-to-date review. Int J Mol Sci. 2017;18(12):2586.

24. Porcaro $G$ et al. Vitamin D plus epigallocatechin gallate: a nove promising approach for uterine myomas. Eur Rev Med Pharmacol Sci. 2020;24(6):3344-51.

25. Zhang $D$ et al. Antiproliferative and proapoptotic effects of epigallocatechin gallate on human leiomyoma cells. Fertil Steril. 2010;94(5):1887-93.

26. Zhang D et al. Green tea extract inhibits proliferation of uterine leiomyoma cells in vitro and in nude mice. Am J Obstet Gynecol. 2010;202(3):281-9.

27. Ahmed RS et al. Biological and mechanistic characterization of novel prodrugs of green tea polyphenol epigallocatechin gallate analogs in 
human leiomyoma cell lines. J Cell Biochem. 2016;117(10):2357-69.

28. Hilário $\mathrm{SG}$ et al. Action of aromatase inhibitor for treatment of uterine leiomyoma in perimenopausal patients. Fertil Steril. 2009;91(1):240-3.

29. Parsanezhad ME et al. A randomized, controlled clinical trial comparing the effects of aromatase inhibitor (letrozole) and gonadotropinreleasing hormone agonist (triptorelin) on uterine leiomyoma volume and hormonal status. Fertil Steril. 2010;93(1):192-8.

30. Song $\mathrm{H}$ et al. Aromatase inhibitors for uterine fibroids. Cochrane Database Syst Rev. 2013;(10):CD009505.

31. Folkerd EJ et al. Aromatase activity in uterine leiomyomata. J Steroid Biochem. 1984;20(5):1195-200.

32. Eldar-Geva T et al. Effect of intramural, subserosal, and submucosal uterine fibroids on the outcome of assisted reproductive technology treatment. Fertil Steril. 1998;70(4):687-91

33. Gambacorti-Passerini $Z$ et al. Trial of labor after myomectomy and uterine rupture: a systematic review. Acta Obstet Gynecol Scand. 2016:95(7):724-34 